Iran Journal of Nursing (IJN)

Vol 32, No. 119, Aug 2019: 41- 51

\title{
Perceived Stress and Contributing Factors in the Elderly with Urinary Incontinence
}

\author{
Hossein Mohammadi ${ }^{1}$, Farideh Bastani ${ }^{2}$
}

\begin{abstract}
Background \& Aim: Recently, lifestyle-related diseases (e.g., chronic diseases) have greatly increased in old age. Cardiovascular diseases, cancers, hypertension, urinary incontinence, hearing loss, musculoskeletal weakness, and mental disorders are highly prevalent among the elderly, imposing certain anxiety and stress on these individuals. Urinary incontinence is a common physical issue in the elderly, which influences their quality of life and causes stress and psychological tension. High stress levels not only affect the quality of life, but also may prevent the use of coping, self-care, and self-efficacy skills and might play a pivotal role in daily life activities and functional independence. Therefore, perceived stress could play a determining role in urinary incontinence as a psychological component. Therefore, there is a need for basic information and a more accurate assessment of this psychological component for accurate planning in order to formulate optimal care interventions in holistic geriatric nursing and provide resident care and support for health promotion and functional capacity increase in the elderly with urinary incontinence and provide educational-counseling strategies to control and manage stress. With this background in mind, the present study aimed to assess perceived stress and the contributing factors in the elderly with urinary incontinence referring to Hasheminejad Hospital in Tehran, Iran in 2017.

Materials \& Methods: This cross-sectional, causal-correlational study was conducted on 125 individuals aged 60-74 years with urinary incontinence referring to Hasheminejad Teaching Hospital (urology and urinary incontinence specialty and sub-specialty medical center) in Tehran. The participants were selected via continuous sampling, and the inclusion criteria were being a young elderly (in the range of 60-74 years based on the WHO classification), no urology surgery in the past three months, no history of malignancy of the urogenital system, urinary tract infection and serious psychiatric disorders (based on medical file), and no cognitive diseases (receiving a score of $\geq 7$ in the abbreviated mental test [AMT] test). Data were collected using the AMT, a demographic characteristics questionnaire, and Cohen's perceived stress scale with acceptable validity and reliability. Data collection was performed during three months via face-to-face interviews (15-20 minutes), and the process continued for three months. A perceived stress scale has been developed to determine the stress of individuals in the face of unpredictable and uncontrollable life events. After completing the demographic characteristics questionnaire and perceived stress scale, each part of the data collection tool was calculated, and data analysis was performed in SPSS version 16 using descriptive statistics (distribution frequency, frequency, mean and standard deviation) and inferential statistics (Scheffe test, analysis of variance, Pearson's correlation coefficient, and linear regression) at the significance level of $\mathrm{P}<0.05$.

Results: The present study was performed on 125 elderlies (58 males and 67 females) with a mean age of $63.92 \pm 2.66$ years. In terms of the level of education, $12 \%$ of the participants were illiterate, whereas $10.4 \%$, $35.2 \%, 24.8 \%, 8 \%$, and $9.6 \%$ had an elementary school diploma, a junior-high-school diploma, a high-school diploma, and a higher academic degree, respectively. Regarding marital status, $72.8 \%$ of the subjects were married while $1.6 \%$ and $25.6 \%$ were single and divorced or widowed, respectively. The mean and standard deviation of perceived stress in the elderly was estimated at $26.4 \pm 61.89$, which showed a low stress level. The frequency distribution and percentage of perceived stress in the studied elderly showed that $56.6 \%$ of the subjects had low perceived stress and $42.3 \%$ had high perceived stress. According to the results, there was a significant relationship between perceived stress and variables of age, level of education, marital status, economic status, and duration of urinary incontinence, in a way that perceived stress was significantly higher in those aged 65 and higher, compared
\end{abstract}

\footnotetext{
1. MS Student in Geriatric Nursing, School of Nursing and Midwifery, Iran University of Medical Sciences, Tehran, Iran

2. Professor, Department of Public Health Nursing and Geriatrics, School of Nursing and Midwifery, Iran University of Medical Sciences, Tehran, Iran (Corresponding author) Tel: 02143651800Ｔ Email: bastani.f@iums.ac.ir
} 
to those below the age of $65(\mathrm{P}<0.001)$. Moreover, perceived stress was higher in widowed participants, compared to married subjects $(\mathrm{P}=0.005)$. Scheffe test results were indicative of higher perceived stress in illiterate elderlies, compared to the other participants $(\mathrm{P}=0.036)$. There was also a significant relationship between economic status and perceived stress, meaning that those with unfavorable economic status had higher perceived stress, compared to those with good economic status $(\mathrm{P}=0.036)$. In addition, there was a significant relationship between stress of the elderly and urinary incontinence, in a way that stress was higher in those who were dealing with urinary incontinence for more than a year $(\mathrm{P}<0.001)$. Therefore, the higher the duration of urinary incontinence in the elderly, the higher their perceived stress. Regression results also showed that perceived stress in the elderly with urinary incontinence for less than one year was 2.84 units lower than in patients with urinary incontinence for more than one year.

Conclusion: According to the results of the study, about half of the participants had high perceived stress levels, and there was a correlation between the mentioned variables and age, level of education, marital status, economic status, and duration of urinary incontinence. In addition, we found a direct significant association between age and perceived stress, meaning that the higher the age of the elderly, the higher their perceived stress. Moreover, stress was significantly higher in the elderly aged 65 years and higher, compared to the elderly aged 60-65 years, and widowed participants experienced significantly higher perceived stress, compared to married subjects. Regarding the level of education, illiterate subjects had higher perceived stress, compared to the other subjects. However, no significant difference was observed at other levels of education. Moreover, those with unfavorable economic status had higher perceived stress levels, compared to those with good economic status. According to the results, stress was higher in the elderly experiencing urinary incontinence for a year or more. Considering that urinary continence affects the daily life of the elderly, planning for the development of community-based nursing interventions, performing educational/counseling strategies for the control and management of stress, and provision of family and social support are recommended in order to enhance the functional capacity of the elderly with urinary incontinence.

Keywords: Perceived Stress, Elderly, Urinary Incontinence

\section{Conflict of Interest: No}

How to Cite: Mohammadi H, Bastani F, Perceived Stress and the Contributing Factors in the Elderly with Urinary Incontinence. Iran Journal of Nursing. 2019; 32(119):41-51.

Received: 11 May 2019

Accepted: 10 Aug 2019 


\title{
استر س درك شده و عوامل مرتبط با آن در سالمندان مبتلا به بىاختيارى ادرارى
}

\author{
حسين محمدى '، فريده باستانى
}

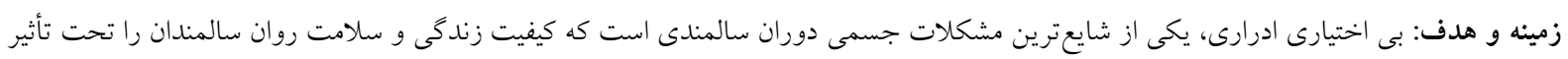

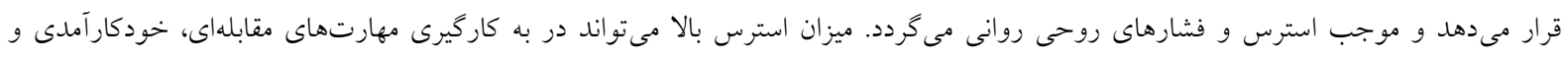

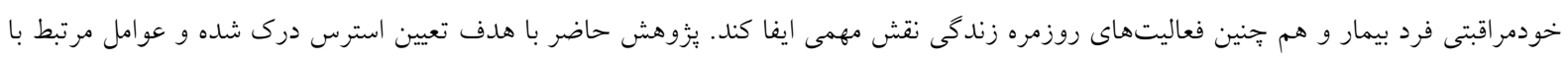

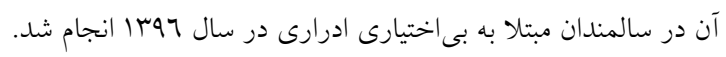

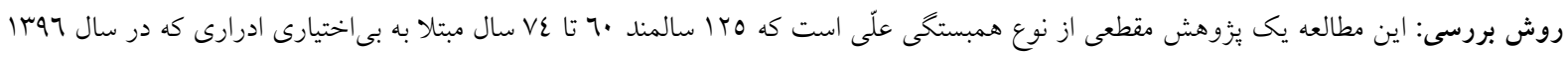

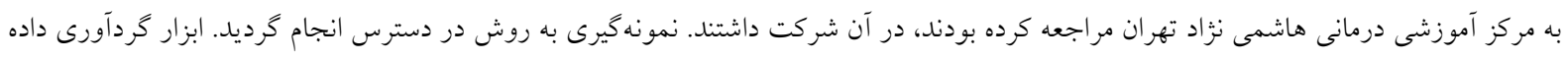

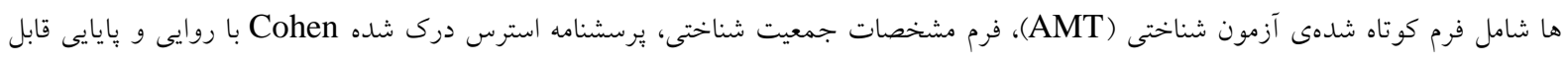

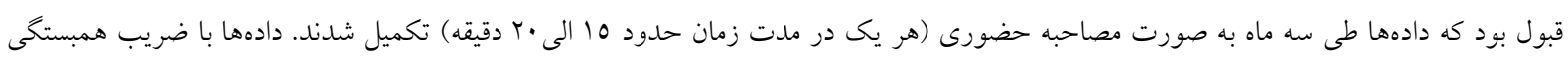

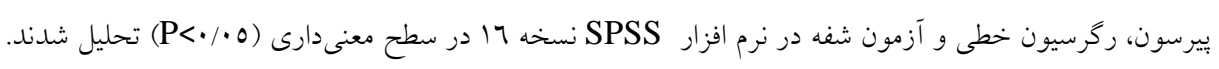

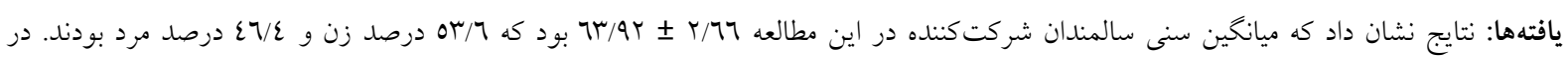

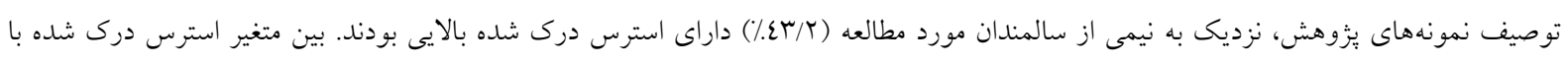

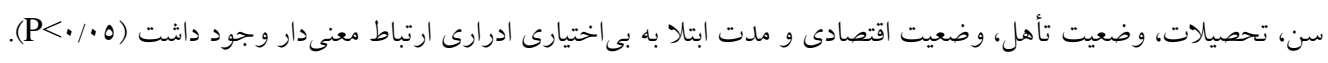

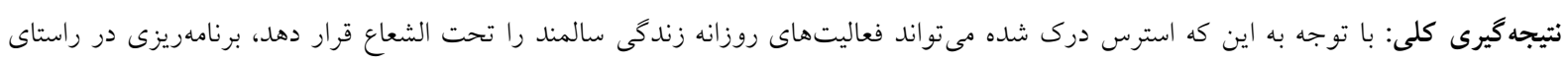

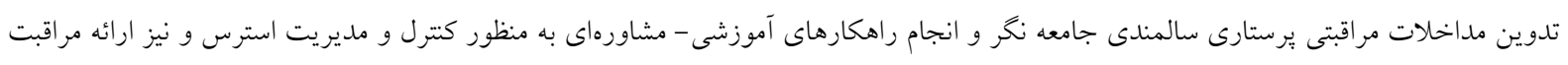

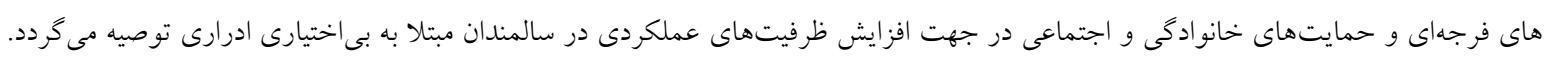
كليد وازهها: استرس درى شده، سالمند، بى اختيارى ادرارى تعارض منافع: ندارد تاريخ دريافت: تاريخ بذيرش: 91/0/19

\footnotetext{
(نويسنده مسئول) ( ) - (ن)
}

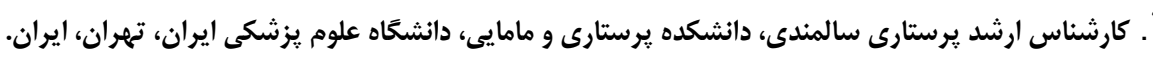

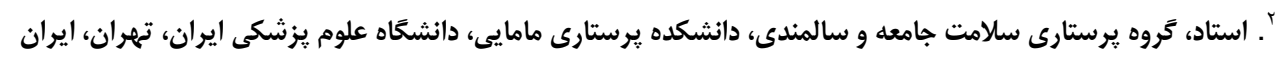


شود("). بر اساس تعريف انجمن بينالمللى بىاختيارى

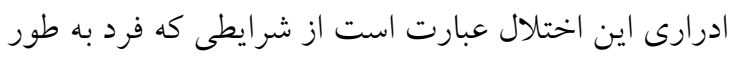

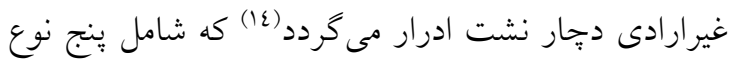

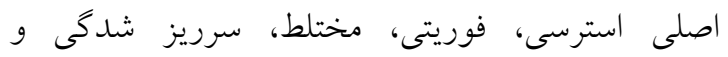

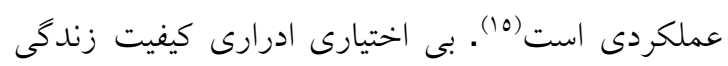

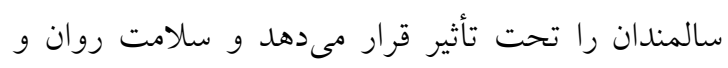

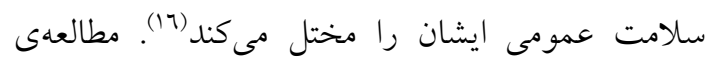
Sumardi اختيارى ادرارى در اندونزى مربوط به سالمندان است. در

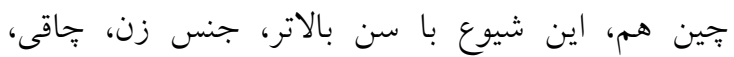

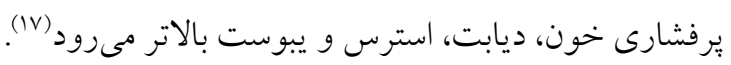
به طور كلى استرس يا فشار روانى نه تنها بر كيفيت زندكى

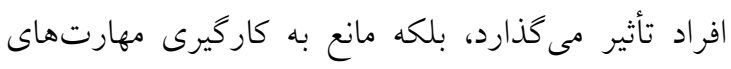
مقابلهاى، خودكارآمدى و خودمراقبتى شده و در فعاليت

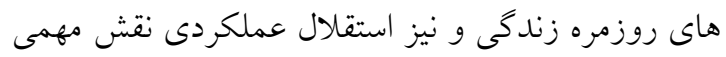

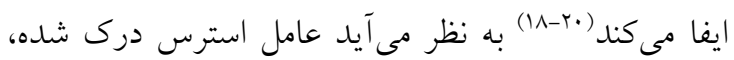

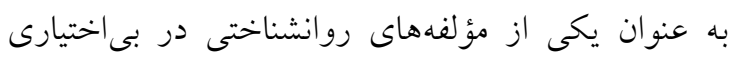

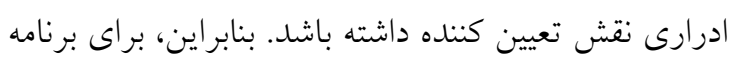

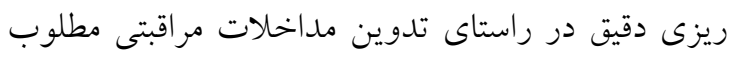

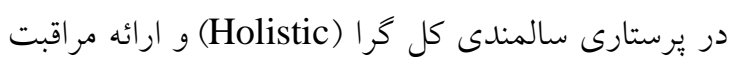
هاى فرجهاى (Respite Care) و حمايتى در خصوص

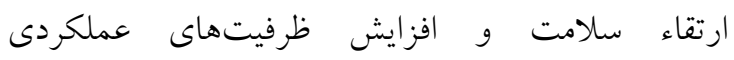
(Functional Capacity)

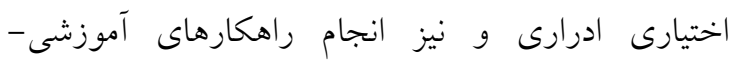

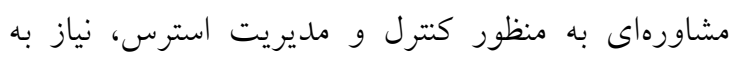
اطلاعات بايهاى و ارزيابى دقيقتر اين مؤلفه روانشناختى وجود دارد. بنابراين هدف از انجام اين مطالعه تعيين استرس درك شده و عوامل مرتبط با آن در سالمندان مبتلا به بى اختيارى ادرارى مراجعه كننده به مركز آموزشى درمانى هاشمى نزاد تهر ان درسال

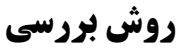

يزّوهش حاضر همبستخى علّى و از نوع مقطعى است.

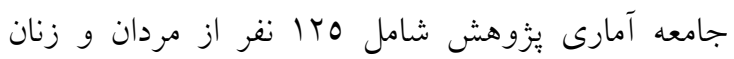
سالمند مر اجعه كننده به مركز آموزشى درمانى هاشمى نزاد

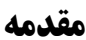

سالمندى بنا به تعريف سازمان جهانى بهداشت، عبور از



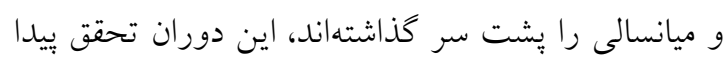
مىكند (1). بر اساس بيش ريش بينى هاى جمعيتى از مركز آمار

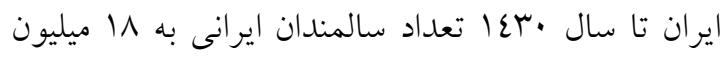

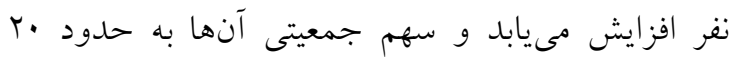

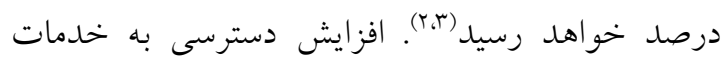
بهداشتى و كاهش بيمارىهاى عفونى و مرى ناشى از آن



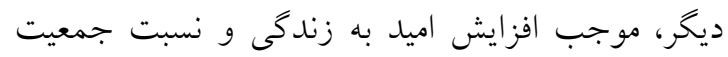
سالمندان شده است. با اين حال، در سالهاى اخير، بيمارى

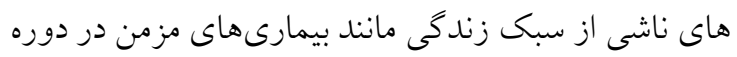

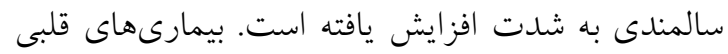
عروقى، سرطانها، برفشارى خون، بى اختيارى ادرارى، بـاني

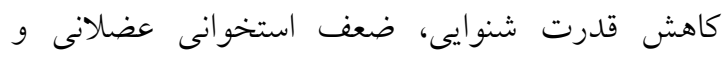

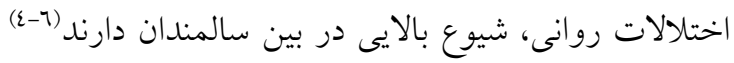
كه اضطراب و استرس خاصى بر سالمند تحميل مى

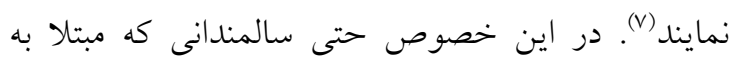

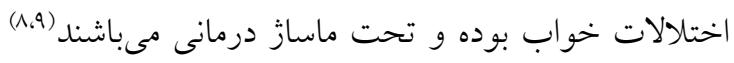

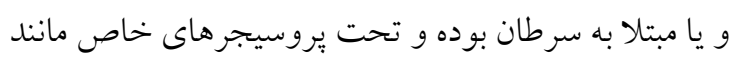

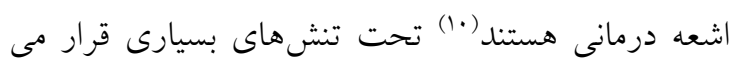
كيرند كه با ارزيابى دقيق ميزان استرس درى درى شده

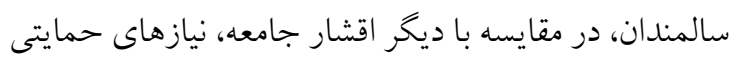

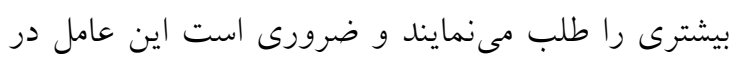
سالمندان با بيمارىهاى خاص مورد ارزيابى قرار كيرد (11).

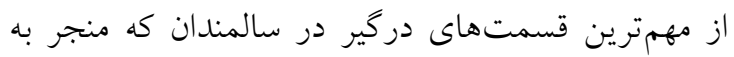

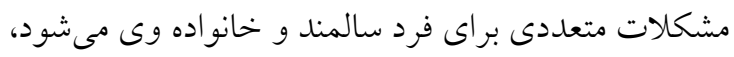
دستخاه ادرارى__تناسلى است. بـىاختيارى ادرارى تمام

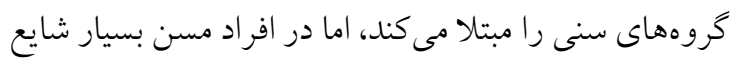

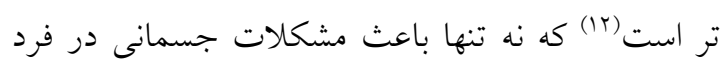
مىشود، بلكه باعث مشكلات روانى، اجتماعى، اقتصادى

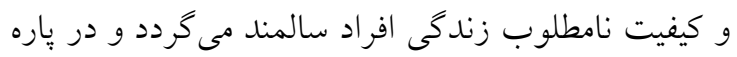
اى موارد منجر به اقامت آنان در خانههاى سالمندان مى 


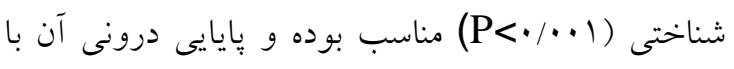

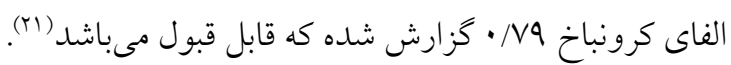
فرم مشخصات فردى يا سئو الات جمعيت شناختى شامل سن، جنسيت، تحصيلات، وضعيت تأهل، وضعيت اقتصادى، تعداد فرزندان، بيمارىهاى همزمان و سئولات والات وصعيت

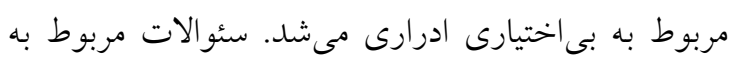

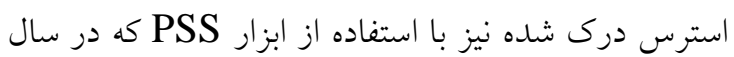

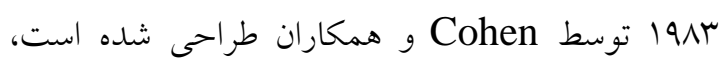
سنجيده شد (Yr). مقياس استرس درى شده جهت تعيين

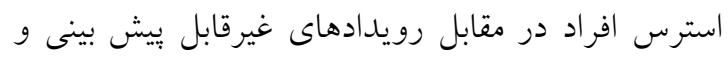

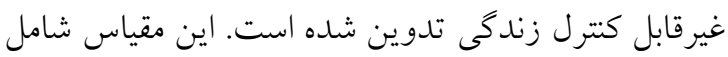

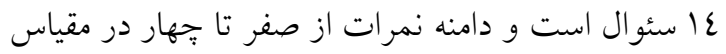

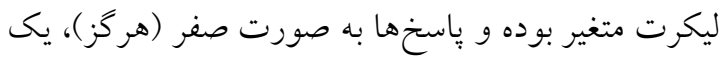
(به ندرت)، دو (كاهى اوقات)، سه (بيشتر اوقات) و جهار

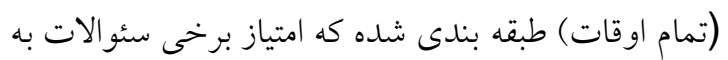
طور معكوس محاسبه مىشود. در كل، دامنه نمرات بين

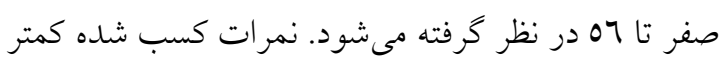

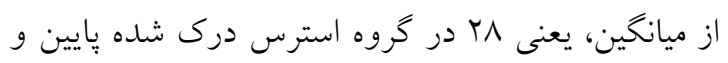

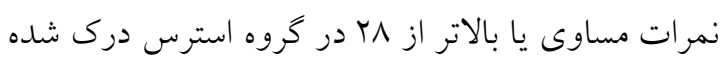

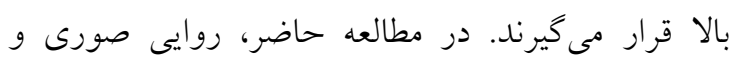
محتواى اين مقياس با نظر سه تن از اساتيد فن و انجام

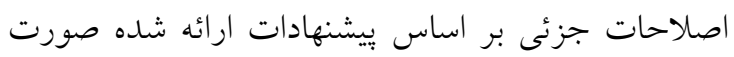

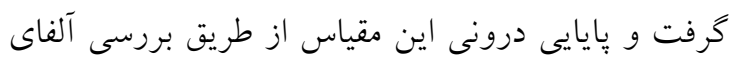

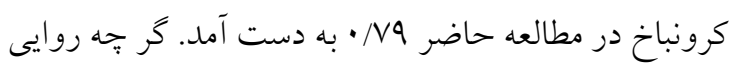
و پايايى علمى اين مقياس در مطالعه يورسيد و همكاران،

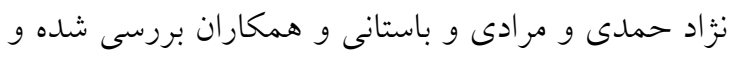

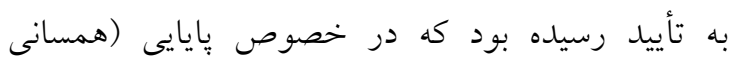

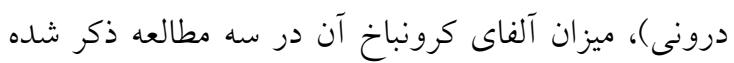

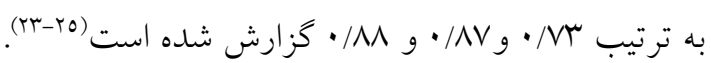
يس از تكميل فرم اطلاعات جمعيت شناختى و مقياس استرس درى شده، هر يك از بخشهاى ابزار كردآورى

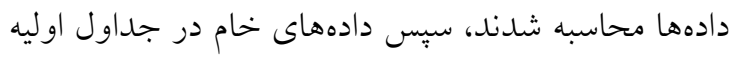

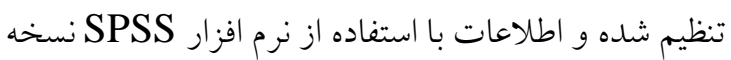
17 و به روش آمار توصيفى (جداول توزيع فراوانى،
تهران (يك بيمارستان تخصصى و فوق تخصصى كليوى و مجارى ادرارى) در سال

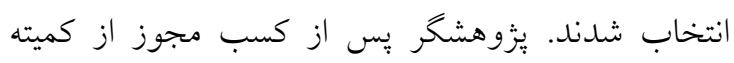

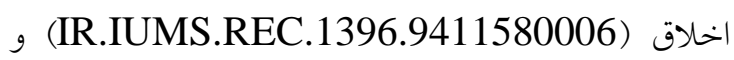
معرفى نامه از دانشخاه علوم يزشكى ايران و يس از توضيح

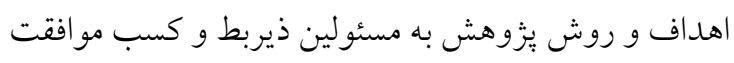

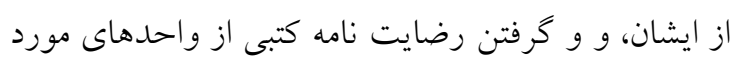

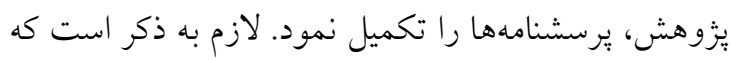

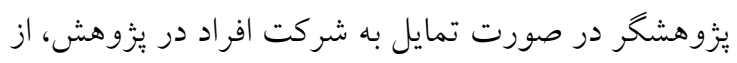

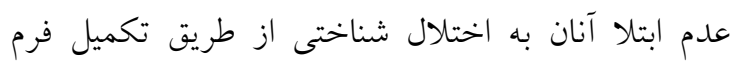
شناختى اطمينان حاصل مىنمود و سبس فرم اطلاعات

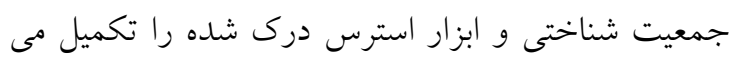
كرد. بر اين اساس، يُزوهشخر با مراجعه روزانه به مركز

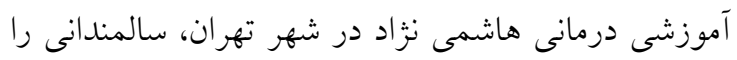

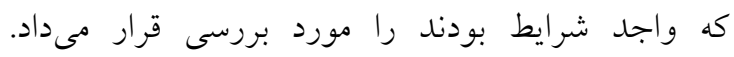

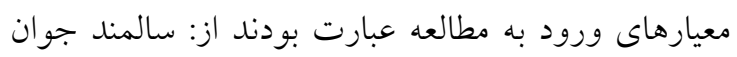

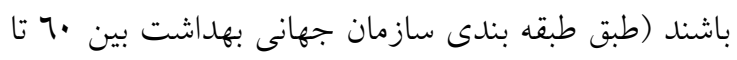

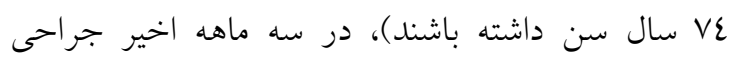

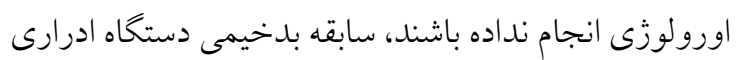

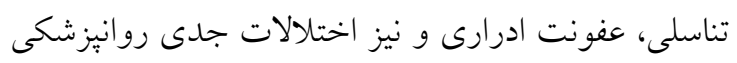

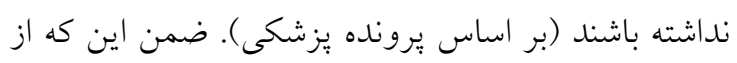

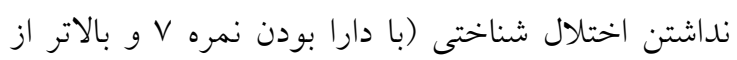
تست AMT) در سالمندان مورد مطالعه نيز اطمينان (آنسان

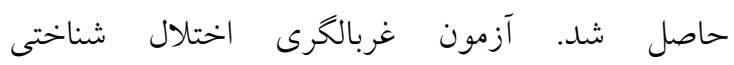
حاوى ده (Abbreviated Mental Test, AMT) سئوال است كه از يركاربردترين آزمونهاى غربالخرى

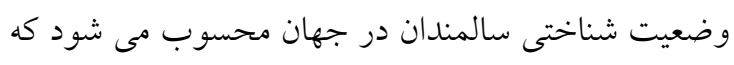

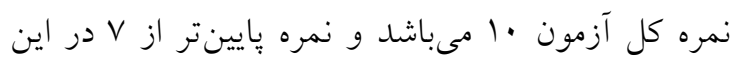

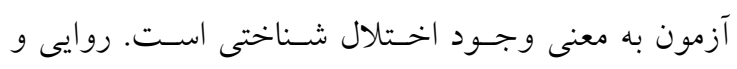

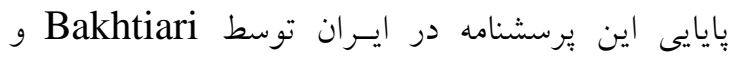

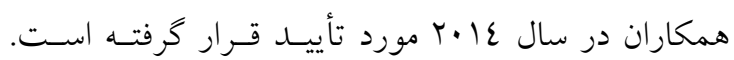
به طورى كه روايى افتراقى آزمون كوتاه شده شناختى براى تشخيص افراد بدون اختلال شناختى يا با اختلال 
جدول شماره ا: توزيع فراوانى و شاخصهاى عددى استرس

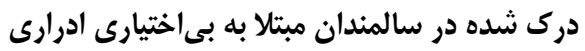

\begin{tabular}{|c|c|c|}
\hline درصد & فراوانى & استرس درك شده \\
\hline $07 / 1$ & VI & 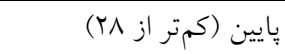 \\
\hline$\varepsilon r / r$ & or & 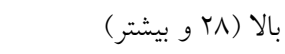 \\
\hline $1 \cdots$ & Iro & جمع \\
\hline \multicolumn{2}{|c|}{$r 7 / \varepsilon \pm 71 / \wedge q$} & انحرف معيار 土 ميانخين \\
\hline \multicolumn{2}{|c|}{$11-r q$} & بيشينه - كمينه \\
\hline
\end{tabular}

بر اساس جدول شماره ا با وجود اين كه ميانخين و انحر اف معيار استرس درك شده در سالمندان مورد مطالعه 1/1/99 توزيع فراوانى و درصد استرس درى شده در سالمندان مورد مطالعه نشان داد 07/7 درصد واحدهاى مورد

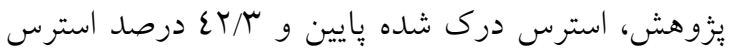
درى شده بالا داشتند،
فراوانى، ميانخين و انحراف معيار) و استنباطى (آزمونهاى

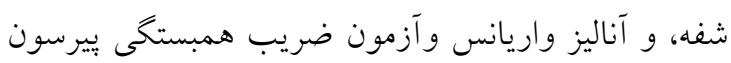
و آناليز رگرسيون) مورد تجزيه و تحليل قرار گرفت.

\section{يافتهها}

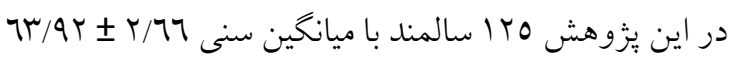
سال مورد مطالعه قرار كرفتند كه ON نفر مرد و TV نفر زن بودند. با درصد بيسواد ع/ • ا درصد داراى سواد خواندن


سيكل، 1 درصد دييلم و 9/7 درصد تحصيلات بالاتر از دييلم داشتند. از ميان سالمندان VY/A درصد متأهل، I/T درصد مجرد و TO/T در درد بيوه و يا همسر فوت شده

بودند

جدول شماره ז: شاخصهاى عددى استرس درى شده در سالمندان مبتلا به بىاختيارى ادرارى بر حسب متغيرهاى جمعيت شناختى

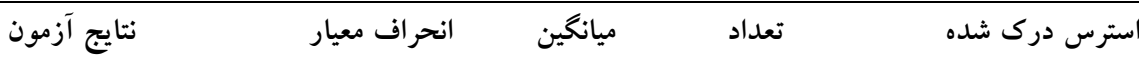

\begin{tabular}{|c|c|c|c|c|c|}
\hline${ }^{*} \mathrm{t}=\mathrm{r} / 91 \mathrm{~d} \quad \mathrm{df}=1 \mathrm{rr}$ & $\varepsilon \pi\urcorner$ & $r T / r V$ & v9 & زير 70 & سن (سال) \\
\hline $\mathrm{P}<\bullet / \cdots 1$ & $0 / .0$ & $r Q / N r$ & $\varepsilon\rceil$ & 7070 & \\
\hline${ }^{*} \mathrm{t}=1 / 7 V r \quad \mathrm{df}=1 \mathrm{rr}$ & $\varepsilon / N V$ & $r \wedge / r q$ & 01 & مرد & جنسيت \\
\hline $\mathrm{P}=. / .9 \mathrm{~V}$ & $\varepsilon / 9 T$ & $r 7 / q \varepsilon$ & TV & 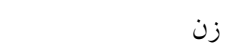 & \\
\hline "F=r/フ7। & $\varepsilon / 7 r$ & $r \cdot / \Lambda T$ & 10 & بيسواد & \\
\hline \multirow[t]{4}{*}{$\mathrm{P}=\cdot / \cdot \mu r$} & $\varepsilon / \varepsilon \wedge$ & $r T / 9 T$ & ir & سواد خواندن نوشتن & \\
\hline & $0 / 11$ & rV/ar & $\varepsilon \varepsilon$ & ابتدايى & تحصيلات \\
\hline & 0 & $\mathrm{rV} / \cdot \mathrm{q}$ & l & سيكل & \\
\hline & 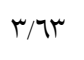 & ro/9 & rr & دييلم و بالاتر & \\
\hline$" \mathrm{t}=\mathrm{r} / \wedge 9 \mathrm{r} \quad \mathrm{df}=|r|$ & $\varepsilon / \varepsilon$ & $r T / \Lambda 1$ & 91 & متأهل & وضعيت تأهل \\
\hline $\mathrm{P}=\cdot / \cdots$ & $0 / 0 \mathrm{~V}$ & $r q / 7 r$ & rt & همسر فوت شده & \\
\hline$* t=\cdot / 79 \quad \mathrm{df}=|r|$ & $\Delta N \varepsilon$ & TN/TV & 11 & $1-r$ & تعداد فرزندان \\
\hline $\mathrm{P}=\cdot / 29 r$ & $\varepsilon / \wedge$ & $r V / \varepsilon)$ & $1 \cdot 0$ & ل ب و بيشتر & \\
\hline$* F=r / 199$ & $\varepsilon / 90$ & $r 7 / \cdot 9$ & rr & خوب & \\
\hline \multirow[t]{2}{*}{$\mathrm{P}=\cdot / \cdot \varepsilon \varepsilon$} & $\varepsilon / 7 \vee$ & rV/70 & $9 \varepsilon$ & متوسط & وضعيت اقتصادى \\
\hline & o/va & $r \cdot / \Lambda$ & 9 & بد & \\
\hline $\mathrm{t}=\varepsilon / \wedge V \quad \mathrm{df}=1 \mathrm{r}$ & $r / \Lambda r$ & TORV & or & كمتر از يك سال & مدت بى اختيارى \\
\hline $\mathrm{P}<\bullet / \cdots+1$ & $\varepsilon / q \mu$ & ra/rr & VI & يك سال و بيشتر & ادرارى \\
\hline
\end{tabular}


بالاترى نسبت به سالمندان با وضعيت اقتصادى خوب

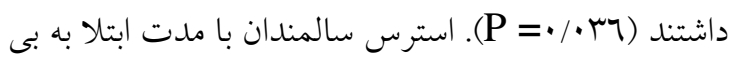
اختيارى ادرار ارتباط معنىدار داشته است. به طورى كه استرس در سالمندانى كه مدت ابتلا به بى اختيارى ادرار آنان يك سال و بيشتر بود، بالاتر گزارش شد ( ( + (P).

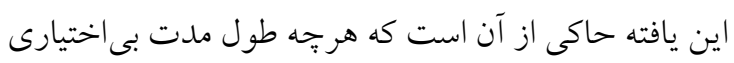
ادرارى در سالمندان بيشتر باشد استرس درى شده بالاترى را تجربه مى كنند. در اين مطالعه، بر اساس جدول شماره ما، نتايج رگرسيون خطى عوامل مؤثر بر استرس درك شده در سالمندان مورد يُزوهش را نشان مىدهد. همان كونه كه ملاحظه مى گردد مدت بى اختيارى ادرارى تنها متغيرى است كه در مدل ركرسيونى معنى دار شد. ملاحظه مىشود استرس درى شده در سالمندان با مدت ابتلا به بى اختيارى ادرارى كمتر

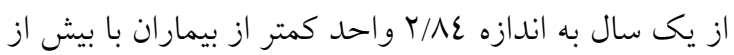
يك سال بى اختيارى ادرارى بود.
در اين يزوهش، جدول شماره Y نشان مىدهد كه متغير هاى سن، تحصيلات، وضعيت تأهل، وضعيت اقتصادى و مدت بى اختيارى ادرارى با استرس درك شده ارتباط معنى دار آمارى داشته است. به طورى كه استرس درى شده در سالمندان 70 سال و بالاتر به طور معنىدارى بيشتر از

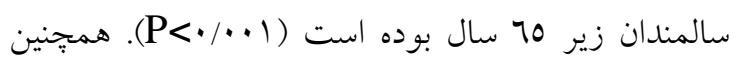
سالمندانى كه همسرشان فوت شده بود نسبت به متأهلين به طور معنى دارى استرس درى شده بالاترى داشتند $(\mathrm{P}=\cdot / \cdot \cdot 0)$ مقايسه دو به دو آزمون شفه براى بررسى آنكه استرس در كدام سطوح تحصيلى با بقيه اختلاف معنى دار آمارى داشته

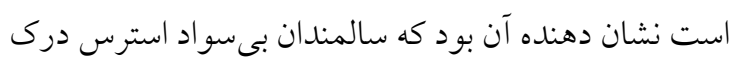
شده بالاترى نسبت به ساير سالمندان داشتند (7س •/ = (P ). وضعيت اقتصادى نيز متغير ديخرى بود كه با استرس ارتباط معنى دار آمارى داشت. مقايسه دو به دو نشان دهنده آن بود كه سالمندان با وضعيت اقتصادى بلد استرس درى شده

\begin{tabular}{|c|c|c|c|c|c|c|}
\hline $\mathbf{R}^{2}$ & $\mathbf{P}$ & $\mathbf{T}$ & ضريب استاندارد & ضر يب & \multicolumn{2}{|c|}{ متغيرهاى مستقل } \\
\hline \multirow[t]{13}{*}{ •/ror } &.$/ .94$ & $1 / 790$ &.$/ 17$ & $\cdot / 491$ & & \\
\hline & & & رده مرجع & & بيسو اد & \\
\hline &.$/ .94$ & $-1 / 9 \cdot \varepsilon$ & $-\cdot / r \cdot 7$ & $-r / r \varepsilon 9$ & سو اد خواندن و نوشتن & \\
\hline & .1 .09 & $-1 / Y \mid r$ & $-\cdot / 17 \mathrm{~V}$ & $-1 / 794$ & ابتدايى & تحصيلات \\
\hline &.$/ T Y \Lambda$ & $-1 / 7 \cdot \varepsilon$ & $-\cdot / r 11$ & $-T / \Gamma \wedge \varepsilon$ & سيكل & \\
\hline &.$/ 111$ & $-1 / \mu r \varepsilon$ &.$- / 1 \mathrm{VO}$ & $-Y / Y I V$ & دييلم و بالاتر & \\
\hline & & & رده مرجع & & همسر فوت شده & وضعيت تأهل \\
\hline & $\cdot / \varepsilon \mid \varepsilon$ & $-/ \Lambda Y$ & $-\cdot / \cdot v \wedge$ & $-\cdot / \Lambda 7 \varepsilon$ & متأهل & \\
\hline & & & رده مرجع & & بد & \\
\hline & $\cdot / 417$ & $-1 / \cdot \cdot 1$ &.$- / 171$ & $-r / \cdot$ rO $_{0}$ & خوب & وضعيت اقتصادى \\
\hline & - & $-\cdot / \varepsilon \vee \wedge$ & $-\cdot / \cdot v \varepsilon$ & $-\cdot /$ Ar & متوسط & \\
\hline & & & رده مرجع & & بيش از يك سال & مدت بى اختيارى ادرارى \\
\hline & $\cdot \cdots r$ & $-r / I r$ & $-\cdot / / 9$ & $-Y / \Lambda \varepsilon Y$ & كمتر از يك سال & \\
\hline
\end{tabular}

همجِون سن، تحصيلات، وضعيت تأهل، وضعيت اقتصادى 
داشتند و بقيه سطوح تحصيلى با يكديكر اختلافى را نشان

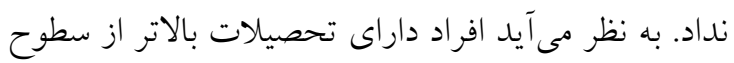

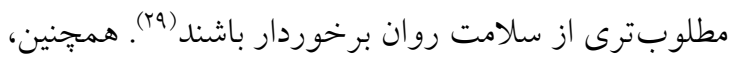

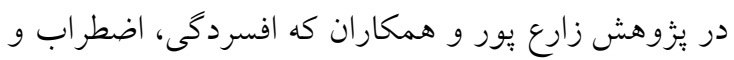

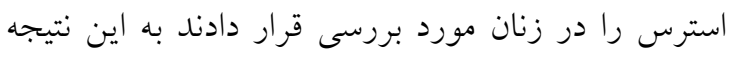
دست يافتند كه متغيرهاى جمعيت شناختى همجيون تحصيلات، ارتباط معنىدار آمارى يا استرس زنان مونيت مورد

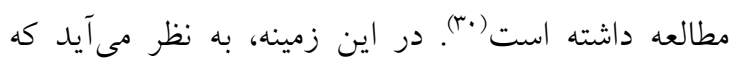
تحصيلات با افزايش اعتماد به نفس افراد مرتبط باشد كه اين امر مىتواند موجب افزايش ارتباطات و تعاملات

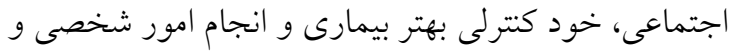

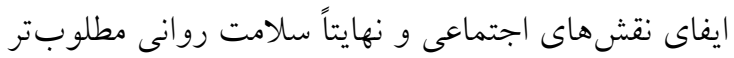

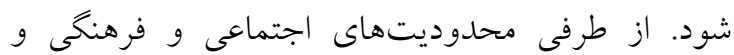

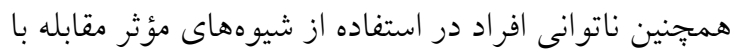
عوامل استرس زا رامىتوان دليل بالاتر بودن استرس در

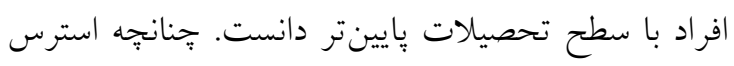

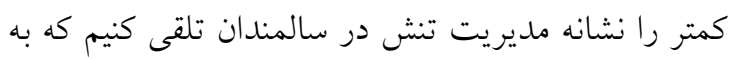
عنوان يك رفتار بهداشتى و ارتقادهنده سلامت مطرح

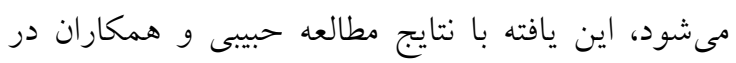

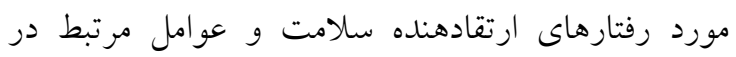

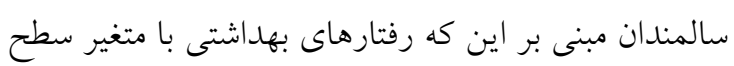
تحصيلات سالمندان ارتباط معنى دار دارد همسو مىباشد.

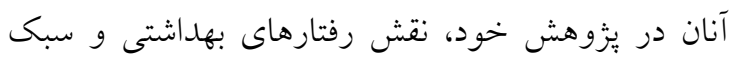

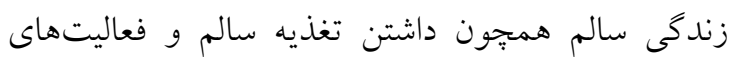
فيزيكى و ورزش و مقابله با استرس را در سالمندان با هـان

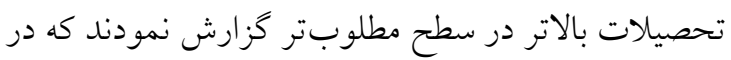

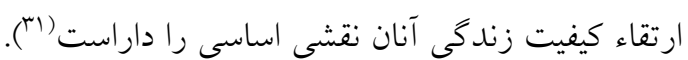

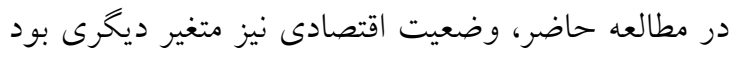
كه با استرس ارتباط داشت. مقايسه دو به دو آزمون شفه نشان داد كه سالمندان با وضعيت اقتصادى بد داراى استرس درك شده بالاترى نسبت به سالمندان با وضعيت

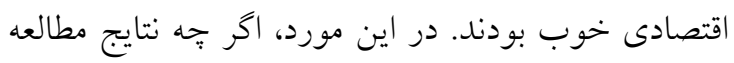

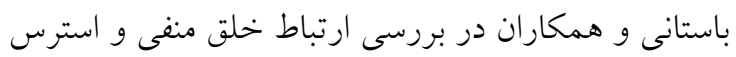
درى شده در زنان نشان داد كه بين وضعيت اقتصادى-
ارتباط مستقيم معنىدار وجود دارد. به عبارت ديخر هر

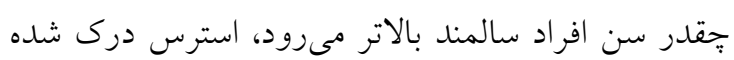

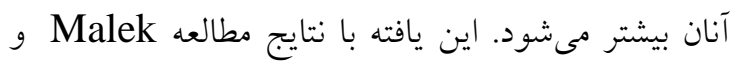

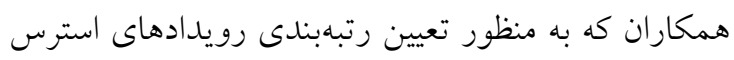

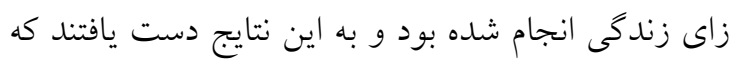

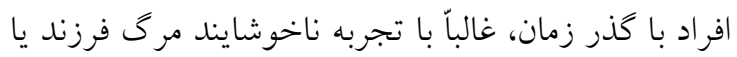

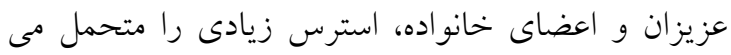
شوند همخو انى دارد. ضمن اين كه متغير "سن " يك عامل تعيينكننده مهم در نمرات استرس افراد به ويزه سالمندان

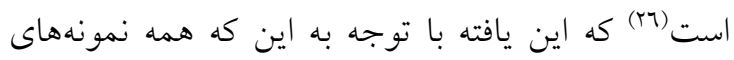
يُزوهش در مطالعه حاضر، افر اد سالمند بودند و درصد قابل

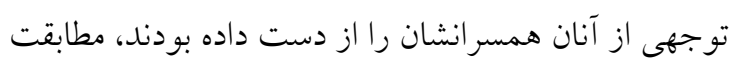

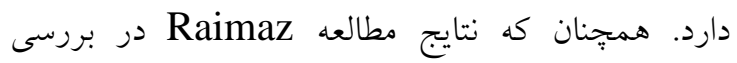
سلامت روان و رابطه آن با سرمايه اجتماعى سالمندان نشان

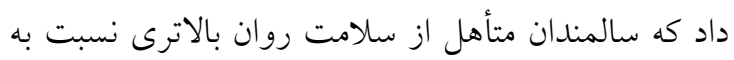

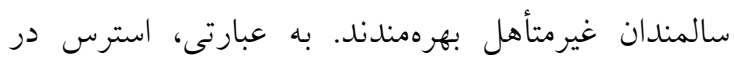

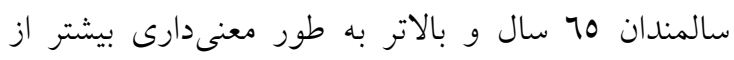

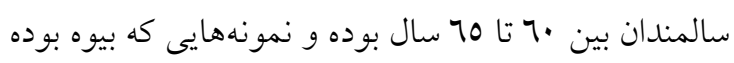
و همسر انشان فوت كرده بودند، نسبت به سالمندان متأهل به طور معنى دارى استرس درك شده بالاترى را تجربه كرده

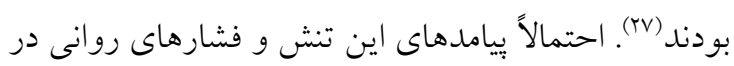

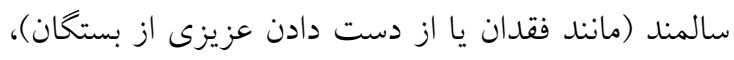
به جز اختلال در سلامت جسمانى، عواقب روانشاختى

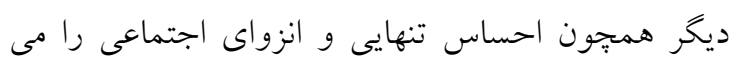
تو اند به همر اه داشته باشد كه به كاهش استقلال عملكردى (functional independency) سالمند به ديخران منجر شده كه مغاير با هدف غائى

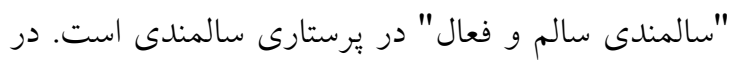

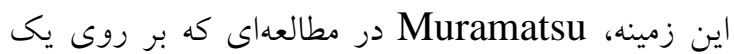
كروه از سالمندان آمريكايى انجام داد دريافت كه سالمندان مجرد، بيوه يا مطلقه سلامت روانى كمترى نسبت به

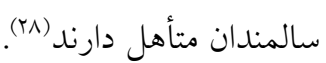
در اين مطالعه، نتايج نشان دهنده آن بود كه سالمندان بيسواد، استرس درك شده بالاترى نسبت به ساير سالمندان 
سالمندى جامعه نخر و انجام راهكارهاى آموزشى - منام

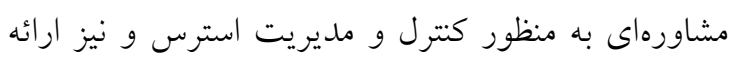

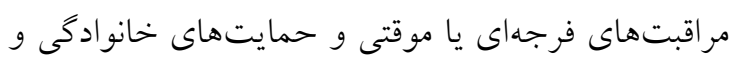

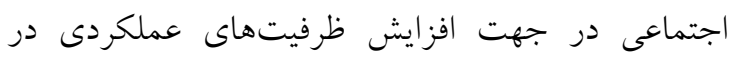

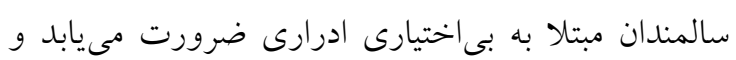
توصيه مى گردد. در اين يززوهش، بيش از نيمى از سالمندان مورد مطالعه

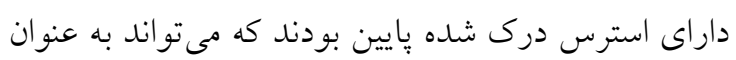
يكى نقطه قوت و فرصت محسوب شود، كه توصيه مى شىود

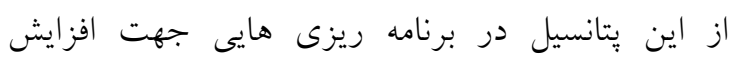

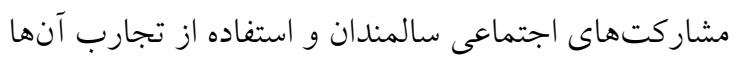

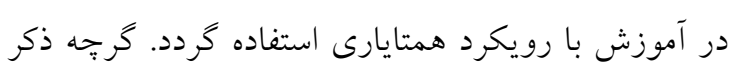

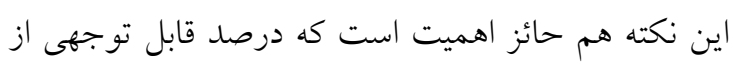

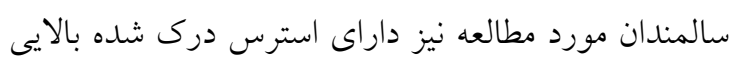

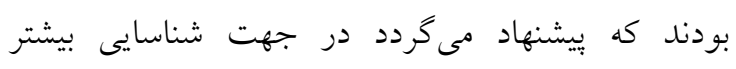
مشكلات و وقايع استرسزا، مطالعهاى كيفى در اين دسته

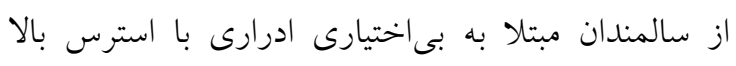

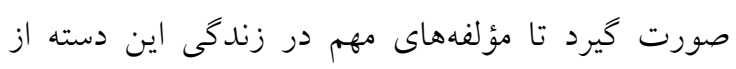
سالمندان مورد واكاوى عميقترى قرار خيرد.

تعارض منافع: هيج گونه تعارض منافع وجود ندارد.

\section{تقدير و تشكر}

از تمامى سالمندان مراجعه كننده به مركز آموزشى درمانى تُّك هاشمى نزاد كه صبورانه با ما همكارى كردند، صميمانه

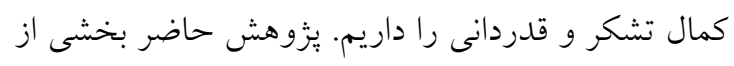

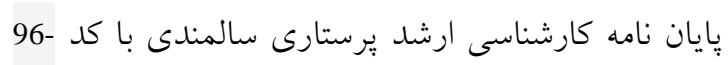

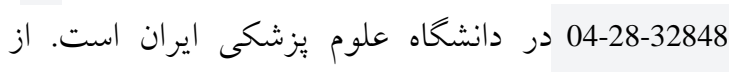

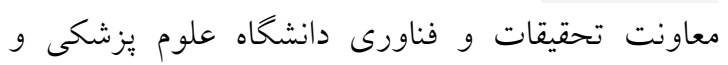
خدمات بهداشتى درمانى ايران كه حامى مالى اين طرح بودند قدردانى مى كردد.
اجتماعى و استرس درك شده افر اد ارتباط معنى دارى وجود ندارد، اما در زنان شاغل نسبت به زنان خدان خانهدار ميانخين

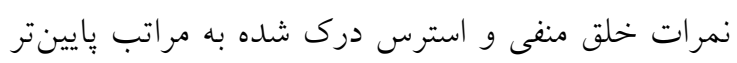

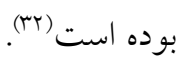

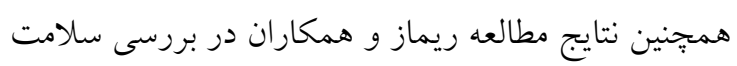

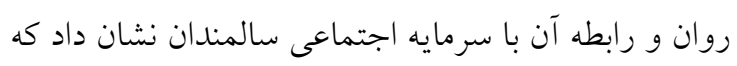

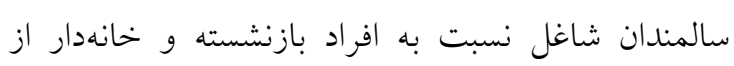

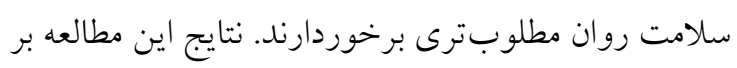

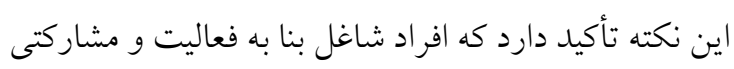
كه در جامعه دارا هستند امكان تعاملات اجتماعى و دخيل

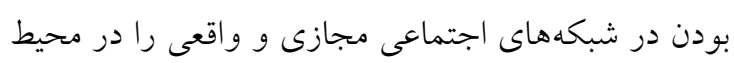

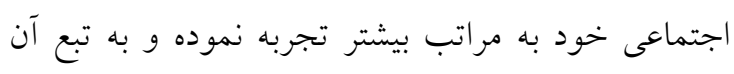

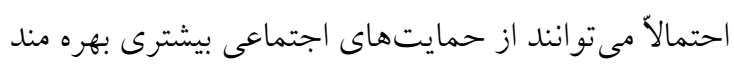

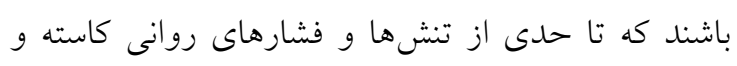

$$
\text { موجب ارتقاء سلامت روان مى خردد (IV). }
$$

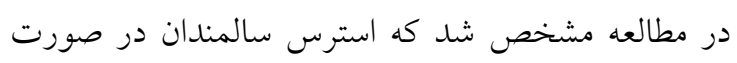
ابتلا آنان به بى اختيارى ادرارى يكى سال و بيشتر، به طور

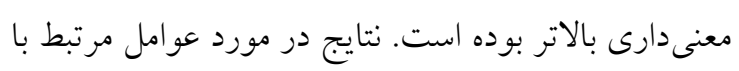
استرس درك شده در سالمندان مورد يزوهش نشان مى مدهد

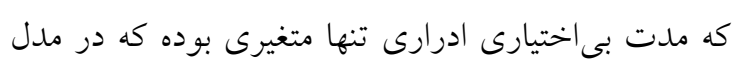

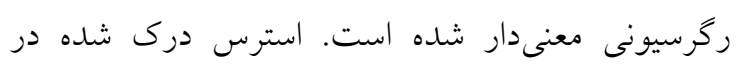

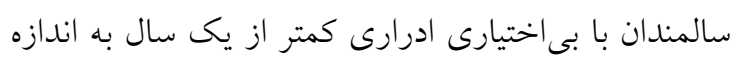

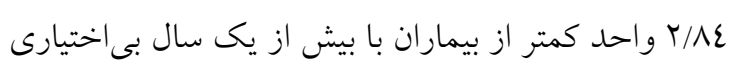

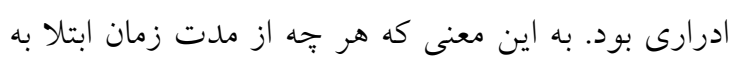

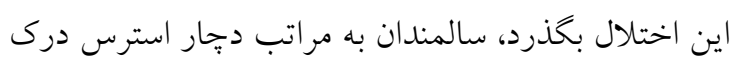
شده بالايى خواهند شد. در اين مطالعه، درصد قابل توجهى از سالمندان مبتلا به بى اهن

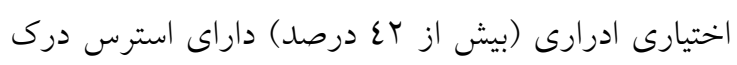

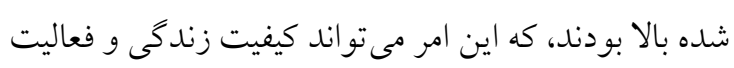

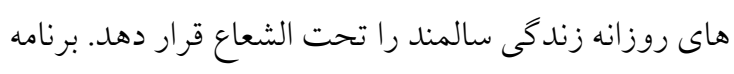

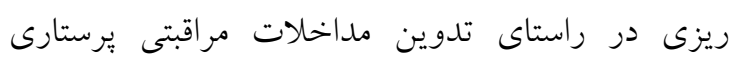




\section{References}

1. Ocampo JM. Self-rated health: Importance of use in elderly adults. Colombia Médica. 2010;41(3):275-89.

2. Statistical Center of Iran. Selected Findings of the 2016 National Population and Housing Census . Vice Presidency Plan and Budget Organization. 2016 Available at: https://www.amar.org.ir/Portals/1/census/2016/Census_2016_Selected_Findings.pdf . Accessed on $3^{\text {rd }}$ Dec 2019

3. Statistical Center of Iran. Islamic Republic of Iran. Vice Presidency for Strategic Planning and Supervision Statistical Centre of Iran, Selected Findings of National Population and Housing Census. 2011 Available at: https://www.amar.org.ir/Portals/1/Iran/90.pdf. Accessed on $3^{\text {rd }}$ Dec 2019.

4. Bastani F, Samady Kia P, Haghani H. The Effect of Inhalation Aromatherapy With Damask Rose (Rosa Damascena) on the Pain of Elderly After Knee Arthroplasty. JCCNC. 2017;3(2):153-60. [Persian]

5. Talebi EL, Bastani FA, Haqhani HA. Effect of Tai Chi Exercise on the Stress of Elderly Women With Hypertension. JCCNC. 2017;3(4):263-8. [Persian]

6. Zeinalhajlu AA, Amini A, Tabrizi JS. Consequences of population aging in Iran with emphasis on its increasing challenges on the health system (literature review). Depiction of Health. 2015;6(1):8. [Persian]

7. Basiri MA, Bastani FA, Haghani HA, Rahmatnejad LE. Effect of Slow-Stroke Back Massage on Anxiety of Older Women With Breast Cancer Undergoing Chemotherapy'. JCCNC. 2016;2(2):11522. [Persian]

8. Abbasi Fakhravari A, Bastani F, Haghani H. The Effect of Foot Reflexology Massage on the Sleep Quality of Elderly Women With Restless Leg Syndrome. JCCNC. 2018;4(2):96-103. [Persian]

9. Banaei A, Hashemi B, Bakhshandeh M, Mofid B. Evaluation of various common prostate IMRT techniques based on estimated tumor control and normal tissue complication probabilities in correlation with patients anatomical parameters derived from the CT scans. Polish Journal of Medical Physics and Engineering. 2019;25(1):35-41.

10. Haghparast A, Hashemi B, Eivazi MT. Influence of compensator thickness, field size, and off-axis distance on the effective attenuation coefficient of a cerrobend compensator for intensity-modulated radiation therapy. Medical Dosimetry. 2013;38(1):25-9.

11. Karamivand VA, Bastani FA, Haghani HA. Support Needs of Urban and Rural Elders in Kermanshah City, Iran. JCCNC. 2018;4(1):21-8. [Persian]

12. Baba Mohammadi $\mathrm{H}$, Khalili $\mathrm{H}$. The effect of pelvic floor exercise in the treatment of urinary incontinence in the elderly, Ghrzy and Imam Reza hospitals in Sirjan city. Yazd Journal of Medical Sciences and Health Services. 2006;3:61. [Persian]

13. Sumardi R, Mochtar CA, Junizaf J, Santoso BI, Setiati S, Nuhonni SA, Trihono PP, Rahardjo HE, Syahputra FA. Prevalence of urinary incontinence, risk factors and its impact: multivariate analysis from Indonesian nationwide survey. Acta Med Indones. 2016;46(3).

14. Borges JB, Neri L, Sigrist RM, Martins LO, Guarisi T, Marchesini AC. Assessing quality of life of women with urinary incontinence using the Kings Health Questionnaire. Einstein. 2009;7:30813.

15. Thirugnanasothy S. Managing urinary incontinence in older people. Bmj. 2010;341:c3835.

16. Sinclair AJ, Ramsay IN. The psychosocial impact of urinary incontinence in women. The Obstetrician \& Gynaecologist. 2011;13(3):143-8.

17. Lai H, Gardner V, Vetter J, Andriole GL. Correlation between psychological stress levels and the severity of overactive bladder symptoms. BMC urology. 2015;15(1):14.

18. Alipour F, Sajadi H, Forouzan A, Nabavi H, Khedmati E. The role of social support in the anxiety and depression of elderly. Iranian Journal of Ageing. 2009;4(11):53-61-.[Persian]

19. Bastani F, Birjandi M, Haghani H, Sobhan MR. Factors Associated With Fear of Falling and Functional Independence in Older Adults in Iranian Nursing Homes. JCCNC. 2016;2(3):135-44. [Persian]

20. Banaei A, Hashemi B, Bakhshandeh M, Mofid B. Trade-off between the conflicting planning goals in correlation with patient's anatomical parameters for intensity-modulated radiotherapy of prostate cancer patients. J Radiother Pract. 2019:1-7. 
21. Bakhtiyari F, Foroughan M, Fakhrzadeh H, Nazari N, Najafi B, Alizadeh M, Arzaghi M, Sharifi F, Shoaee S, Mostafa Q. Validation of the persian version of Abbreviated Mental Test (AMT) in elderly residents of Kahrizak charity foundation. Iranian Journal of Diabetes and Metabolism. 2014;13(6):487-94. [Persian]

22. Cohen S, Kamarck T, Mermelstein R. A global measure of perceived stress.. 1983 1:385-96.

23. Behroozi N, Shahani Yeylaq M, Pourseye S M. Relationship between perfectionism, perceived stress and social support with academic burnout. Strategy For Culture. 2013;5(20):83-102. [Persian]

24. Ahmadi N, Moradi A. The relationship between mindfulness, coping strategies, perceived stress and life quality of cancer patients. J New Researchers on Psychology. 2014;9:32-6.

25. Bastani F, Pourmohammadi A, Haghani H. Relationship between perceived stress with spiritual health among older adults with diabetes registered to the association of diabetes prevention and control in Babol, 2013. Journal of hayat. 2014;20(3):6-18. [Persian]

26. Malek A, Dadashzadeh H, Poorafkary N. Ranking of stressful life events in general population of Tabriz-Iran. Medical Journal of Tabriz University of Medical Sciences and Health Services. 2008 21;30(12):73-80. [Persian]

27. Rimaz S, Abolghasemi J, Seraji S. The relationship of different dimensions of social support with older adults' quality of life in the 8th district of Tehran in 2013. J Educ Community Health. 2015 15;2(1):29-37. [Persian]

28. Muramatsu N, Yin H, Hedeker D. Functional declines, social support, and mental health in the elderly: Does living in a state supportive of home and community-based services make a difference?. Social Science \& Medicine. 2010 Apr ;70(7):1050-8.

29. Harpham T, Grant E, Rodriguez C. Mental health and social capital in Cali, Colombia. Social science \& medicine. 2004;58(11):2267-77.

30. Zareipour M, Sadaghianifar A, Amirzehni J, Parsnezhad M, Ayuoghi Rahnema V. Exploring of depression, anxiety and stress in pregnant women referred to health centers of Urmia. Rahavard Salamat Journal. 2017;3(2):19-30. [Persian]

31. Habibi A, Nikpour S, Seyedoshohadaei M, Haghani H. Health promoting behaviors and its related factors in elderly. Iran journal of nursing. 2006;19(47):35-48. [Persian]

32. Bastani F, Hidarnia A, Montgomery KS, Aguilar-Vafaei ME, Kazemnejad A. Does relaxation education in anxious primigravid Iranian women influence adverse pregnancy outcomes?: a randomized controlled trial. The Journal of perinatal \& neonatal nursing. 2006;20(2):138-46. [Persian] 